\title{
Radiative Heat Transfer on Turbulent Mixed Convection Flows
}

\author{
T. Anvesh, R. Harish
}

\begin{abstract}
In the present study our intention is to dispatch the turbulent mixed convective flow and radiative flow in a vertical rectangular channel. The channel is constructed with two openings inlet and outlet. The turbulence is modelled by computational fluid dynamics (CFD) approach using lambremhorst turbulence model. Radiation is modelled with Discrete ordinates method (DOM). Finite difference method (FDM) is utilized to discretize the governing equations and an inhouse Fotron code is used to simulate the turbulent flow. The invariant study is carried out for the effect of flow opposite to buoyancy force and flow in same direction as buoyancy flow heat transfer characteristics. The heat transfer rate is remarkably altered by the flow opposite to buoyancy force and flow in same direction as buoyancy flow behavior observed inside the enclosure

Keywords: lambremhorst Turbulence, Assisting flow, Opposing flow, discrete ordinates method.
\end{abstract}

\section{INTRODUCTION}

This Many Analytical and Numerical experiments have done for easy understanding of fluid and heat transfer process in the vertical channels. Mixed convection is a combination of free and forced convection. Natural convection or free convection is an existing energy in the daily life. In short natural convection in any fluid motion is caused by natural means such as buoyancy effect. In forced convection the flow is forced to flow over a surface or in a channel. Tkachenko et.al [1] performed analytical study in a vertical rectangular channel and found that common convective air entering the channel changing with the wall emissivities on the channel wall having Hot wall toward one side and cold wall on the opposite end discovered complex three dimensionless unsteady vortical structures. These structures began to shape ahead of schedule up stream and direct contact with the stream and warmth move in the channel. Andreozzi and Manca[2] performed analytical study by assessing the average Nusselt number by methods for analytical information. Correlations for the Nusselt number, globally averaged at the midpoint of Nusselt number and Rayleigh number are assessed. Andreozzi et. al[3] performed analytical study on the unsteady common convection in air, channel-chimney

Revised Manuscript Received on April 25, 2020.

* Correspondence Author

T. Anvesh, Student, CAD/CAM, School of Mechanical Engineering, Vellore Institute of Technology Chennai, Tamilnadu-600127, India.

R.Harish*, Assistant Professor, School of Mechanical Engineering, Vellore Institute of Technology Chennai, Tamilnadu-600127, India. Email: harish.r@vit.ac.in

(c) The Authors. Published by Blue Eyes Intelligence Engineering and Sciences Publication (BEIESP). This is an open access article under the CC BY-NC-ND license (http://creativecommons.org/licenses/by-nc-nd/4.0/) system was done and made a correlation among the greatest wall temperatures shows that Simple channel is the most basic design in the consistent state condition. To finish up expanding the Ra value the ideal B/b value, as far as Nusselt number, diminishes and terrible arrangement is gotten at higher $\mathrm{B} / \mathrm{b}$ value. Bianco et. al[4] performed an analytical study on air natural convection in a joined channel, with proportionate warming was accomplished. Flow visualization is completed to show the distinctive example of stream between plates in numerous setups Nusselt number and Dimensionless greatest temperatures are assessed and corresponded to Rayleigh number. Li Dong et. al[5] performed conjugate laminar regular convection heat move and wind current with the radiation of solar oriented beneficiary with glass window are numerically examined. The effect of surface emissivity, heating temperature, convective coefficient and temperature of condition on heat move from recipient with glass window is likewise been worked. Akiyama and Chong[6] numerically investigated the interaction of natural convection with warm radiation by grey surfaces in a square enclosure loaded up with air has been worked. The outcomes demonstrated that surface radiation changed the temperature dispersion and flow patterns particularly at higher Rayleigh number. The average convection Nusselt number increments with the expansion of Rayleigh number ( $\mathrm{Ra}$ ). Andreozzi et. al[7] performed numerical study of a heated vertical parallel plate channel owing an assistant plate at inlet and two insulated augmentation plates connected to the heated walls at exit has been completed. The optimal channel configuration communicated regarding highest averaged Nusselt number is for the pre-assigned Grashof number. Florio[8] performed studies were thermal exchanges between particles, liquid and stream ways incorporate particle and particle strong body contact are incorporated. This strategy is applied to an eleven fixed particle design with the stream driver qualities and restricted radiative outflows differed. This strategy improves abilities for investigation of warm collaboration in the particle loaded high speed gas stream and temperature dependent for comprehensive simulation. Kogawa et.al[9] has performed tests in cubic cavity. LES was directed vreman model was received to dynamic sub-grid model. To isolate the radiation impacts of gas and surface radiation for computation condition have analyzed non-radiation, gas radiation, surface and combined radiation and discovered surface radiation was predominant in age of shear stress by turbulent flow. Yao et. al[10] worked on a unified computational frame- work dependent on the Lattice Boltzmann method(LBM) is applied to solve the coupled heat transfer during stage change process with natural convection and volumetric radiation.

Published By:

Blue Eyes Intelligence Engineering \& Sciences Publication

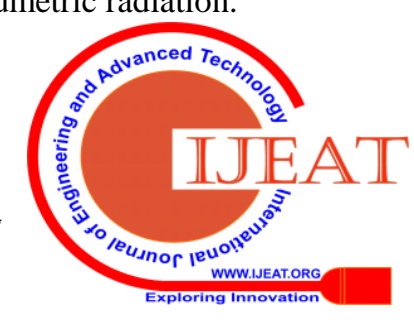


The outcomes by LBM agree well with the benchmark arrangement or other numerical arrangement which shows the model utilized right now of high accuracy and adaptability to recreate coupled heat transfer in multi-dimensionless participating medium. The objective of this study is to work on the turbulent mixed convective flow and conjugate heat transfer characteristics inside the heated vertical channel. The heat source is at left wall and the other end is cold wall and the remaining walls are adiabatic. The problem is solved by solving the continuity equation for mass conservation and the momentum equation for capturing the velocity field and the energy equation for the temperature distribution. Invariant studies have done this by comparing flow opposite to buoyancy force and flow in same direction as buoyancy The Fotron 90 code based Finite difference method was developed to solve governing differential equations

\section{MATHEMATICAL MODELING AND NUMERICAL METHOD}

The vertical rectangular channel taken in our study is having $\mathrm{H}, \mathrm{W}, \mathrm{B}$. There are two openings at the top and bottom of the channel for air flow, and the hot wall is considered to the left and the cold wall is on the right. The remaining walls are considered adiabatic. Generally, adiabatic is nothing but there is no heat is gained or lost by the system. Numerical simulations are performed by using Finite Difference Method (FDM). The Problem is worked in two cases 1 . Assisting case and 2. Opposing case as shown in the figure 1

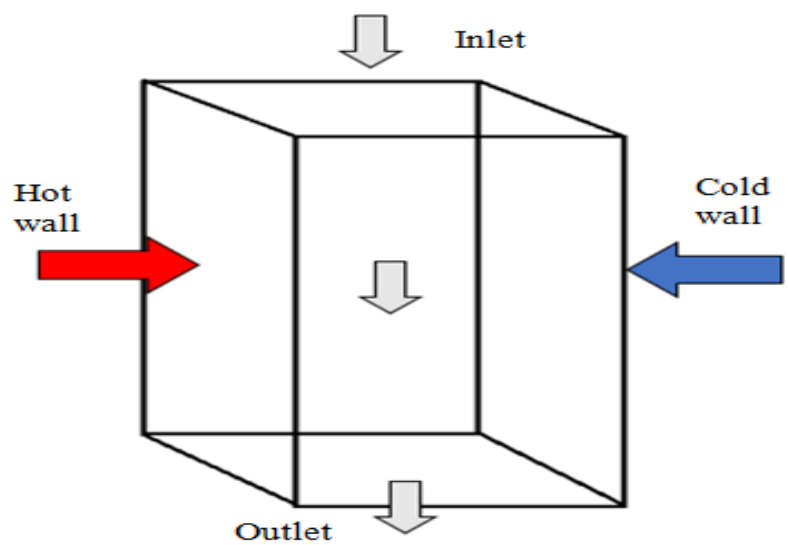

Figure.1. Assisting flow

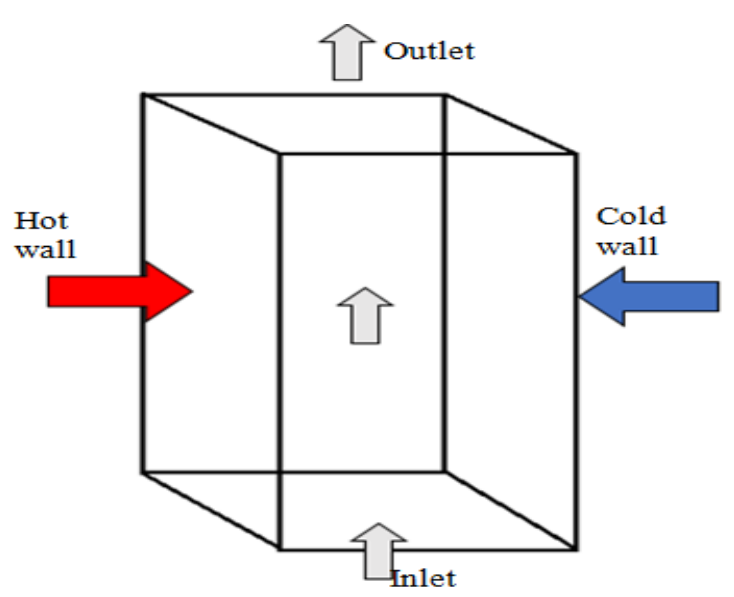

Figure.2. Opposing flow

This study is based on computational fluid dynamics approach using lambremhorst turbulence model. Radiative intensities are evaluated using discrete ordinates method (DOM). It converts radiative transport equation (RTE) into set of partial differential equations. The Radiation transport equation is solved in every step of computational domain to obtain the radiation intensity The turbulent flow of the problem is formulated by solving the Reynolds average Navier - Stokes equations (RANS) for the velocity flow and the energy equation for the temperature field. The study uses a simplified marker and cell algorithm (SMAC) for pressure-velocity decoupling. The time average governing equations are as follows :

$$
\begin{aligned}
& \frac{\delta p}{\delta \mathrm{t}}+\frac{\delta\left(\rho \mathrm{u}_{\mathrm{j}}\right)}{\delta \mathrm{x}_{\mathrm{j}}}=0 \text { (1) } \\
& \frac{\delta\left(\rho \mathrm{u}_{\mathrm{i}}\right)}{\delta \mathrm{t}}+\frac{\delta\left(\rho \mathrm{u}_{\mathrm{i}} \mathrm{u}_{\mathrm{j}}\right)}{\delta \mathrm{x}_{\mathrm{j}}}=\frac{-\delta \mathrm{p}}{\delta \mathrm{x}_{\mathrm{i}}}+\frac{\delta \sigma_{\mathrm{ij}}}{\delta \mathrm{x}_{\mathrm{j}}}+\frac{\delta \mathrm{M}_{\mathrm{ij}}}{\delta \mathrm{x}_{\mathrm{j}}}+\left(\mathrm{\rho}-\mathrm{\rho}_{\mathrm{ref}}\right) \\
& \rho \mathrm{c}_{\mathrm{p}} \frac{\delta \mathrm{T}}{\delta \mathrm{t}}+\frac{\delta\left(\rho \mathrm{c}_{\mathrm{p}} \mathrm{u}_{\mathrm{i}} \mathrm{T}\right)}{\delta \mathrm{x}_{\mathrm{j}}}=\frac{\delta}{\delta \mathrm{x}_{\mathrm{j}}}\left(\mathrm{k} \frac{\delta \mathrm{T}}{\delta \mathrm{x}_{\mathrm{j}}}\right)+\frac{\delta \mathrm{q}_{\mathrm{j}}}{\delta \mathrm{x}_{\mathrm{j}}}+\mathrm{S}_{\mathrm{r}}(3) \\
& \xi_{j} \frac{\delta I_{j}}{\delta x}+\eta_{j} \frac{\delta I_{j}}{\delta y}+\zeta_{j} \frac{\delta I_{j}}{\delta z}=k_{\mathrm{a}}\left(E_{b}-I_{j}\right)(4)
\end{aligned}
$$

The strength of the heat source is denoted by a dimensionless number called Grashof number (Gr). The Grashof number is a dimensionless number, named after Franz grashof. The grashof number is defined as the ratio of the buoyant force to viscous force acting on a fluid in the velocity boundary layer. When the Grashof number increases Thermal expansion following temperature difference increases and Kinematic viscosity decreases. The Grashof number is represented as follows

$\mathrm{G}_{\mathrm{r}}=\frac{g \beta \Delta \mathrm{TL}^{\mathrm{a}}}{v^{2}}(5)$

Were,

$\beta=\frac{1}{\left(T_{h}+T_{\infty}\right)+273(k)}$

For foreseeing the patterns in a fluid's behaviour a dimensionless number is used called as Reynolds number $\left(\mathrm{R}_{\mathrm{e}}\right)$. The Reynolds number is a dimensionless number that gives a measure of the ratio of inertial forces to viscous forces. The Reynolds number is mathematically represented as follows

$\mathrm{R}_{\mathrm{e}}=\frac{\rho v \mathrm{l}}{\mu}$

Were,

$\rho$ is the density, $v$ is velocity, 1 is the length, $\mu$ is viscosity. As Grashof number and Reynolds number are dimensionless we can change the characteristics by altering the Richardson number

$\mathrm{R}_{\mathrm{i}}=\frac{\mathrm{G}_{\mathrm{r}}}{(\mathrm{Re})^{2}}$

Boundary conditions for the vertical rectangular channel is velocity inlet and pressure outlet

\section{RESULT AND DISCUSSION}

Published By:

Blue Eyes Intelligence Engineering

\& Sciences Publication

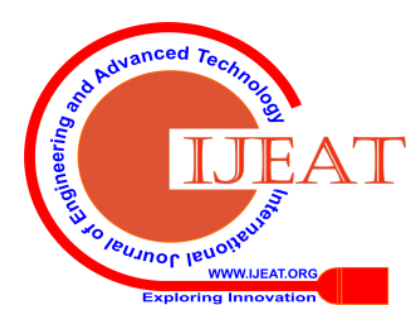


Figure 1 and 2 indicates the flow opposite to buoyancy force and flow in same direction as buoyancy cases considered in the present study. In the present study turbulent mixed convective air enters into the channel were free and forced air enters into the channel as per the respective cases were free air goes in the assisting flow and forced air flow in the opposing flow due to gravitation acting opposite to the direction of flow

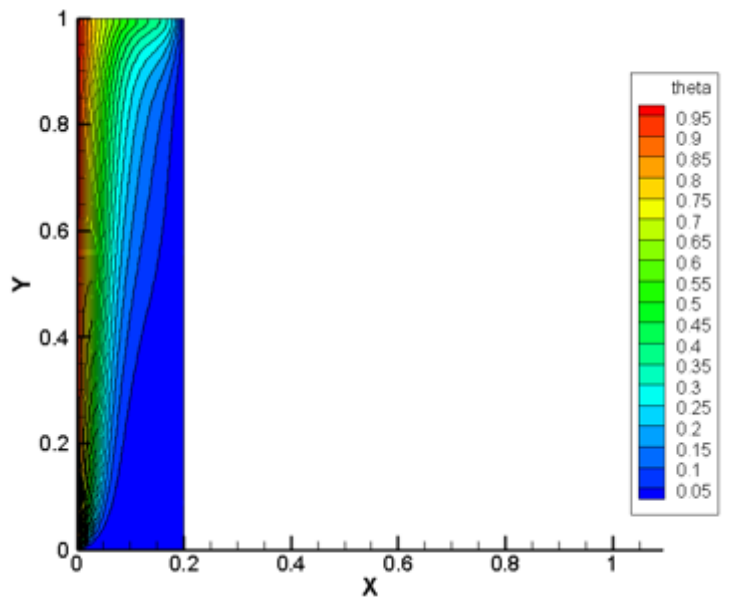

Figure. 3. Temperature contours of Assisting flow For $\mathrm{Gr}=10^{6}$

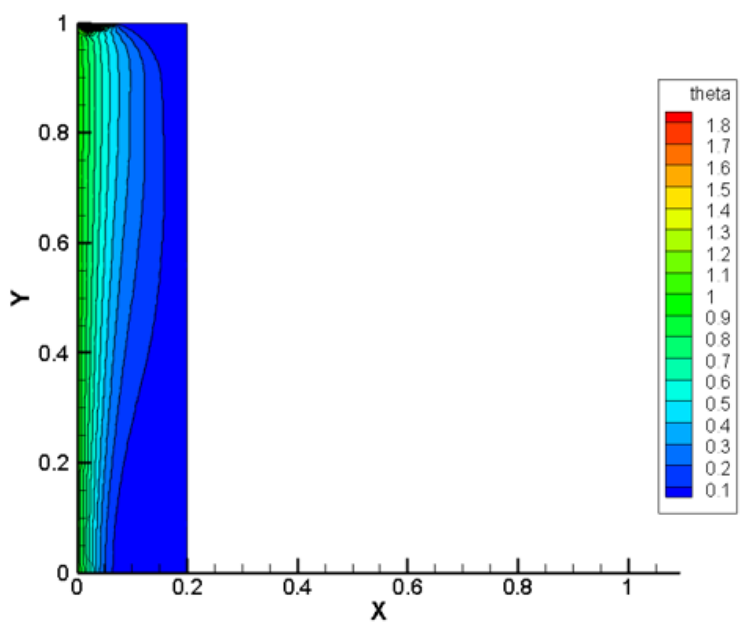

Figure. 4. Temperature contours of Opposing flow for

$$
\mathrm{Gr}=10^{6}
$$

Figure. 3 and 4 indicates evolution of temperature contours inside the enclosure for $(\mathrm{Gr})$ of $10^{6}$. As in the assisting case the turbulent free convective air enters into the channel, we can see the temperature drop in the flow. The flow enters into the channel at $\operatorname{Re}=10^{2}$
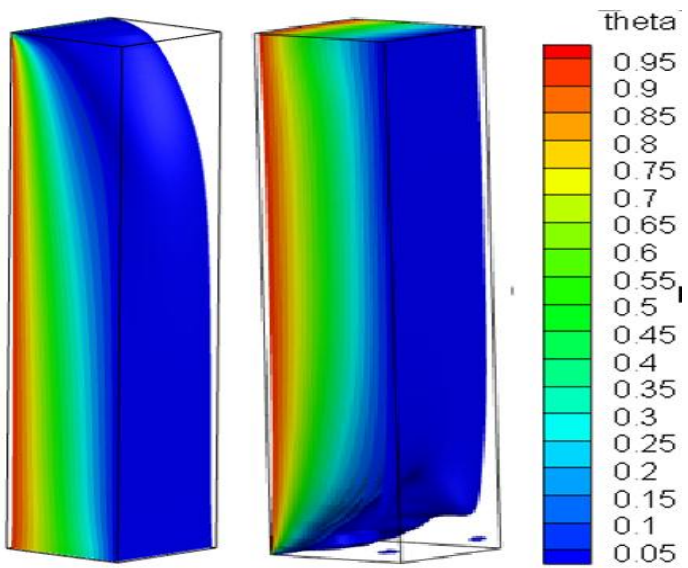

Retrieval Number: C6424029320/2020@BEIESP
Figure. 5. Temperature contours of flow opposite to buoyancy force and flow in same direction as buoyancy flow in 3-D

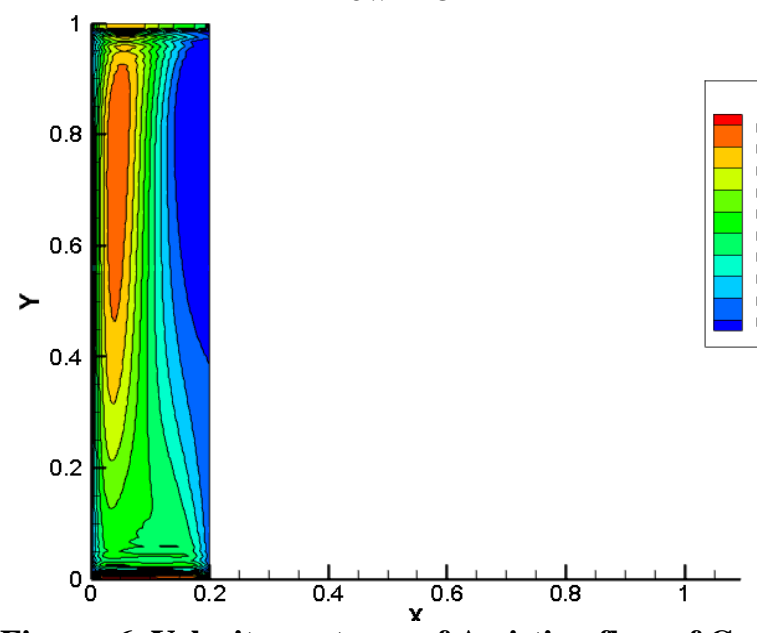

Figure. 6. Velocity contours of Assisting flow of $\mathrm{Gr}=10^{6}$ and $\operatorname{Re}=10^{2}$

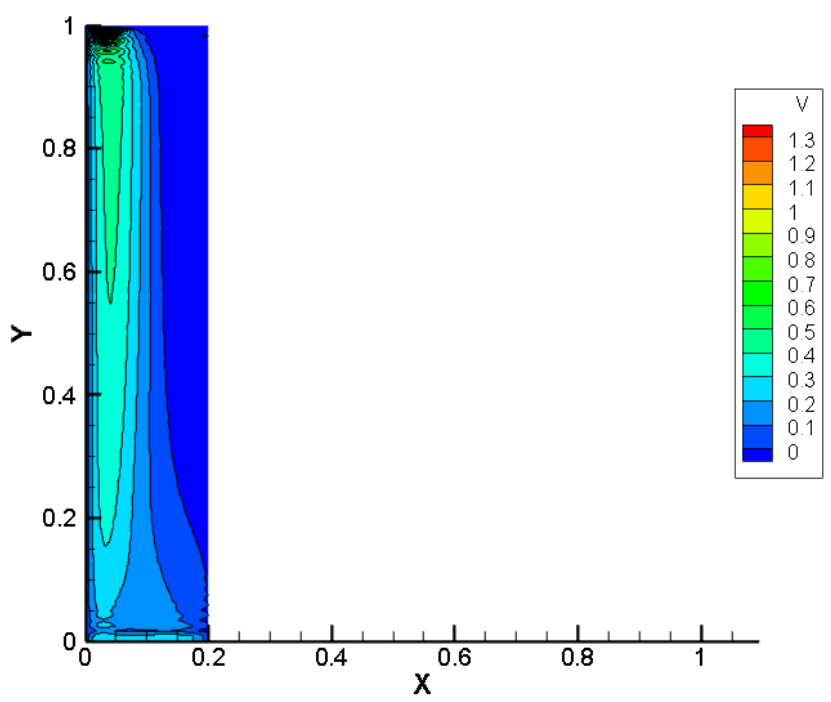

Figure. 7. Velocity contours of Opposing flow of $\mathrm{Gr}=10^{6}$ and $\operatorname{Re}=10^{2}$

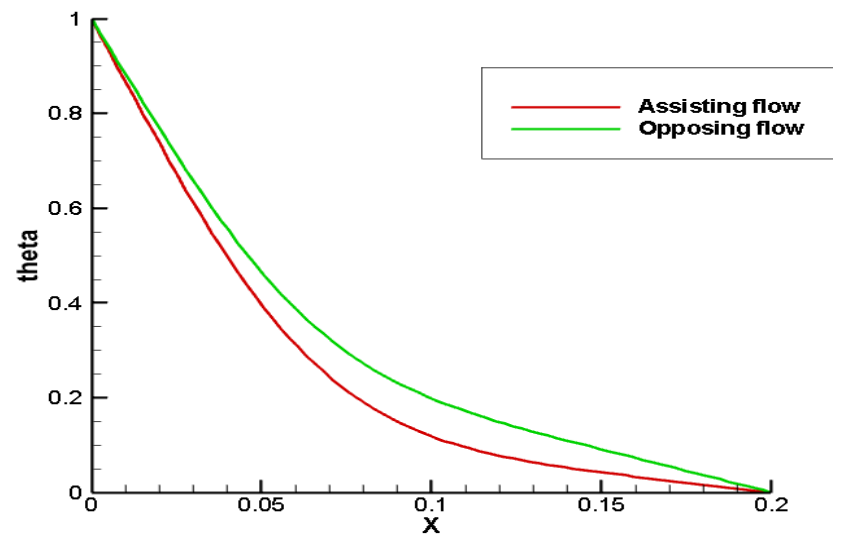

Figure. 8. Temperature distribution of Flow opposite to buoyancy force and flow in same direction as buoyancy flow

Published By:

Blue Eyes Intelligence Engineering \& Sciences Publication (C) Copyright: All rights reserved. 
In the above graph it is evident that temperature is less in the Assisting flow compare to the Opposing flow. The flow in the opposing case has to overcome the gravitation to surpass the vertical channel. So the temperature has increased which is proportional to the pressure which also increases

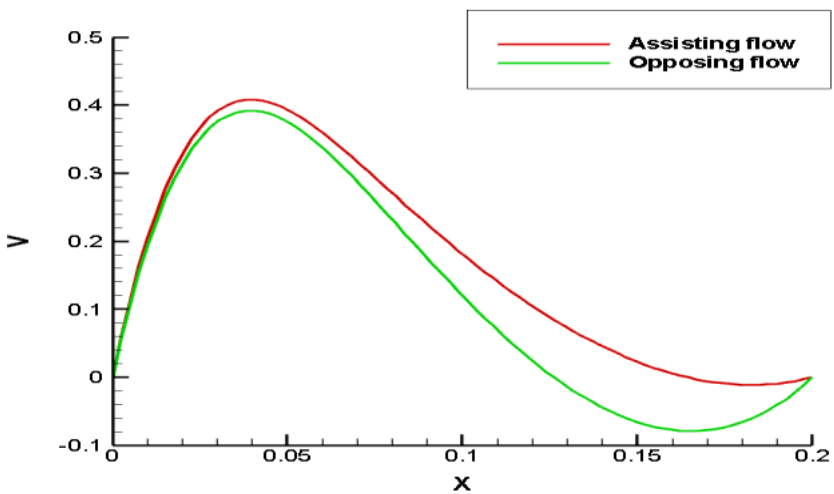

Figure. 9. Velocity distributions of Flow opposite to buoyancy force and flow in same direction as buoyancy flow

In the above graph it is evident that the Assisting case is having high velocity because the turbulent air is taken with the flow of gravitation, were in the Opposing case the velocity of the flow is been oppositely influenced by the gravitation, so the velocity has been decreased

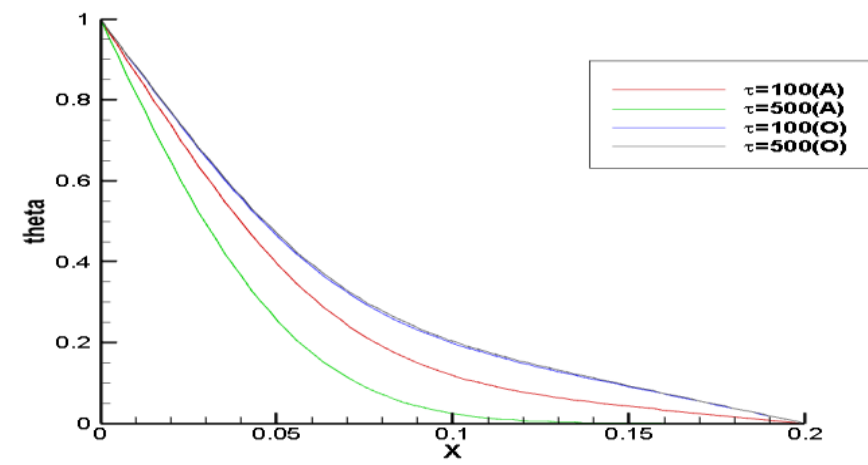

Figure. 10. Temperature distribution of Flow opposite to buoyancy force and flow in same direction as buoyancy flow

In the above graph Flow opposite to buoyancy force and flow in same direction as buoyancy flows of Temperature has been taken into axis. We can observe in the assisting flow as the flow passes from inlet through outlet there is a temperature drop of 0.6 in the horizontal axis has been reduced. when in the Opposing case the temperature is increased slightly when the flow passes from inlet to outlet.

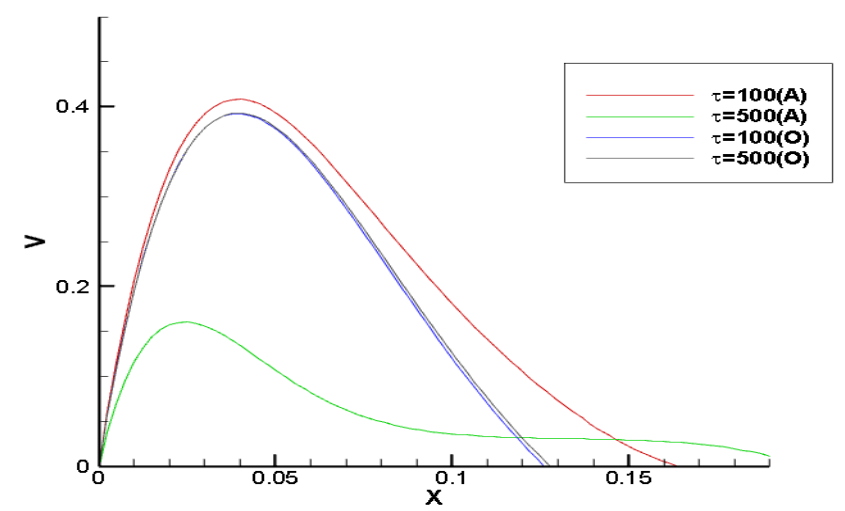

Figure. 10. Velocity distribution of Flow opposite to buoyancy force and flow in same direction as buoyancy flow
In the above graph we can observe there is a sudden drop in the velocity in assisting flow when the turbulent air passes from inlet to outlet, were as in the opposing flow there is 0.01 increases in the velocity were the flow passes from inlet to outlet.

\section{CONCLUSION}

The combined convection of turbulence within a vertical rectangular channel with a hot and cold wall within the channel is solved numerically by changing the flow and its direction. The unstable flow is customized by the CFD approach using the Lambremhorst $\mathrm{k}-\varepsilon$ and turbulence model and is controlled using the finite difference method (FDM). The velocity and temperature are varied by changing the Grashof number and Reynolds number. radiation is modelled by Descrete Ordinates Method(DOM). Along the channel both the temperature and velocity is distributed with respect to time as the flow is unsteady. When comparing both Flow opposite to buoyancy force and flow in same direction as buoyancy flows, there is a difference in assisting flow but where as in Opposing flow there is not much of a difference in velocity and temperature as well. Taking mid plane as consideration there is around $30 \%$ of difference between flow opposite to buoyancy force and flow in same direction as buoyancy flow

\section{REFERENCES}

1. Tkachenko, S., V. Timchenko, G. Yeoh, and J. Reizes. 2019. "Effects of Radiation on Turbulent Natural Convection in Channel Flows.' International Journal of Heat and Fluid Flow 77: 122-133.

2. Andreozzi, Assunta, and Oronzio Manca. 2015. "Radiation Effects on Natural Convection in a Vertical Channel with an Auxiliary Plate." International Journal of Thermal Sciences 97: 41-55.

3. Andreozzi, Assunta, Bernardo Buonomo, and Oronzio Manca. 2012. "Numerical Investigation of Transient Natural Convection in a Vertical Channel-Chimney System Symmetrically Heated at Uniform Heat Flux." International Journal of Heat and Mass Transfer 55 (21-22): 6077-6089

4. Bianco, Nicola, Luigi Langellotto, Oronzio Manca, and Sergio Nardini. 2010. "Radiative Effects on Natural Convection in Vertical Convergent Channels." International Journal of Heat and Mass Transfer 53 (17-18): 3513-3524.

5. Guojun, Zhang, Liu Changyu, and Li Dong. 2018. "Numerical Analysis of Natural Convection and Radiation for Tube Solar Receiver With Glass Window." Journal of Solar Energy Engineering 140 (6).

6. M. Akiyama \& Q. P. Chong (1997) NUMERICAL ANALYSIS OF NATURAL CONVECTION WITH SURFACE RADIATION IN A SQUARE ENCLOSURE, Numerical Heat Transfer, Part A Applications: An International Journal of Computation and Methodology, 32:4, 419-433

7. Andreozzi, Assunta, Antonio Campo, and Oronzio Manca. 2008. "Compounded Natural Convection Enhancement in a Vertica Parallel-Plate Channel.” International Journal of Thermal Sciences 47 (6): 742-748.

8. Florio, L.a. 2019. "Conduction, Convection, and Radiation Thermal Exchange with Discretized Fixed, Contacting Particles in a High Speed Compressible Flow.” International Journal of Heat and Mass Transfer 133: $137-153$.

9. Kogawa, Takuma, et al. "Influence of Radiation Effect on Turbulent Natural Convection in Cubic Cavity at Normal Temperature Atmospheric Gas." International Journal of Heat and Mass Transfer, vol. 104, 2017, pp. 456-466.

10. Yao, Feng-Ju, et al. "Analysis of Melting with Natural Convection and Volumetric Radiation Using Lattice Boltzmann Method." International Journal of Heat and Mass Transfer, vol. 112, 2017, pp. 413-426.

Published By:

Blue Eyes Intelligence Engineering \& Sciences Publication

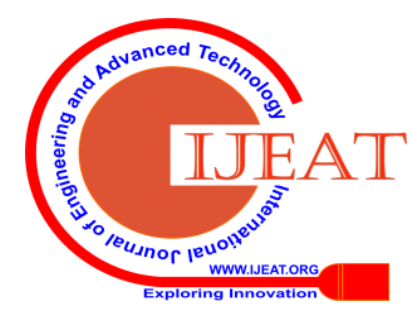




\section{AUTHORS PROFILE}

T. Anvesh is pursuing as an Post Graduate at VIT University Chennai campus. And completed Graduation from SCSVMV University. His research interests are in the field of computational fluid dynamics, Heat Transfer, Turbulent flows and Aerodynamics

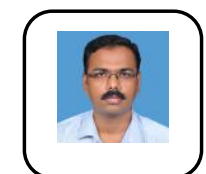

Dr. R. Harish is working as an Assistant Professor at VIT Chennai campus in the school of Mechanical and Building Sciences. His research interests are in the field of computational fluid dynamics, Heat Transfer and Turbulent flows

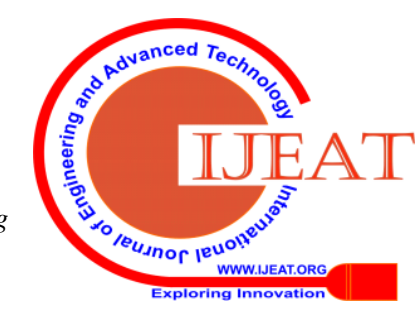

